\title{
The Functions of Continuous Processes in Contemporary Electronic Dance Music
}

\author{
Jeremy W. Smith
}

NOTE: The examples for the (text-only) PDF version of this item are available online at: https://www.mtosmt.org/issues/mto.21.27.2/mto.21.27.2.smith.php

KEYWORDS: EDM, dance music, popular music, deadmau5, form, climax, narrative, function

ABSTRACT: This article discusses the functions of continuous processes in contemporary electronic dance music (EDM), providing an analytical framework for discussing their structural and aesthetic roles in this repertoire. Continuous processes are musical gestures with continuous changes to musical parameters, rather than discrete, "step-by-step" ones. Examples include pitch slides (glissandos), crescendos, fade ins, accelerandos, and filter sweeps. Continuous processes in this repertoire are created with "continuous controllers" such as sliders and knobs, or are programmed into tracks with "automation curves." Functionally, continuous processes often provide sonic instructions for dancers. They can be used by creators at strategic times to provide ornamentation, orientation (often accompanied by intensification or de-intensification), or disorientation (usually in breakdown sections). This article adds to existing analytical scholarship by drawing attention to the many roles of continuous processes in EDM, and showing how they contribute to the emotional waves experienced when listening to this music.

DOI: $10.30535 / \mathrm{mto} .27 .2 .0$

Received August 2020

Volume 27, Number 2, June 2021

Copyright $@ 2021$ Society for Music Theory

[1.1] Producers and fans of electronic dance music (EDM) describe the experience of listening to this repertoire as like riding emotional waves. The superstar producer Armin van Buuren, who works in the EDM genre of trance, says that "Trance music is all about release. It's about energy. It's about emotion ... It's like a wave. You're a surfer."(1) Similarly, producer Rick Snoman, author of the influential Dance Music Manual, says that all dance music "deals mostly in emotional waves, consisting of building and dropping the arrangement to generate an emotional state in the audience" (Snoman 2009, 269). These "emotional waves" of tension and release occur in virtually all forms of music, but they are manifested in specific ways in contemporary EDM and EDMinfluenced pop songs. ${ }^{(2)}$ How do dancers know when the waves are coming, and how to "ride" them? What musical components participate in these narratives to create varying degrees of energy and tension in EDM?

[1.2] Some aspects of tension and release in EDM are caused by what I call discrete processes. For example, Mark Butler states that EDM listeners correlate tension and energy levels with texture changes $(2006,221)$. Tension is linked with the withholding of the beat and specifically the kick drum. The presence or absence of this low drum sound, and its entrance or exit from the texture, is highly important in determining the formal function of different sections in the track and their 
tension levels (Butler 2006, 91, 247). Both Butler and Stan Hawkins recognize metric irregularity and metrical dissonance as another source of tension (Butler 2001; 2006, 138-75; Hawkins 2003, 9294). Polyrhythms, syncopation, and displaced rhythms are all very important in EDM and contribute to its complexity and intensity throughout various tracks. Another common technique that increases tension in "buildup" sections is the snare-drum "roll" or "fill," when percussive sounds such as snare-drum hits become closer and closer together until it almost sounds like a drum roll (Snoman 2009, 252, 266-68; Solberg 2014, 70). The rhythm for a typical snare roll is shown in Example 1. All three of these techniques can be described as discrete processes, since they involve the sudden entrance or exit of a sound layer, or the repetition of discrete sounds in a stratified texture. ${ }^{(3)}$ The snare roll is an example of discrete acceleration, since the "speeding up" takes place in various stages of rhythmic diminution, such that the rhythmic values become twice as fast in each new stage.

[1.3] However, continuous processes also contribute significantly to the emotional waves in EDM tracks. By continuous processes I mean musical gestures produced with continuous changes to musical parameters. ${ }^{(4)}$ Examples include pitch slides (glissandos), crescendos, fade ins, accelerandos, filter sweeps (which continuously change the cutoff threshold of frequencies that are being filtered out of sounds), and timbre changes (accomplished through continuous manipulation of sound envelopes and/or frequency content). ${ }^{(5)}$ Some abstract visual representations of continuous and discrete processes are shown in Example 2. ${ }^{(6)}$

[1.4] This article discusses the functions (that is, the musical roles) of continuous processes in contemporary EDM, focusing on tracks from the 2010s in the genres of trance, big room, progressive house, and electro house. ${ }^{(7)}$ I argue continuous processes are sonic instructions that help dancers ride the emotional waves by serving functions of ornamentation, orientation (which often coincides with intensification or abatement of sonic energy), or disorientation (often in "breakdown" sections). After providing an overview of the scholarship on continuous processes in EDM, popular music, and electronic music generally, as well as a contextualization of how they are created, I will analyze the continuous processes in several tracks and discuss their functions, which are usually based on their location within the form of a track.

\section{How Continuous Processes are Discussed and Created}

[2.1] In EDM and contemporary popular music more generally, continuous processes are not usually considered to be defining features of a "work," but only of a particular text (a fixed musical object such as a recording) or performance. ${ }^{(8)}$ They are often broadly categorized as "effects." For example, Hawkins (2003) refers to "effects processing" (90), "phasing effects" (91), "studio effects (filtering)" (95), "heavy echo effects" (96), and "special effects" (98) in his analysis of "French Kiss" by Lil' Louis. He also describes "gradual manipulation of tempo" and a gradual "working up of the textures" without discussing these in detail (Hawkins 2003, 94). The term "effects processing" references a device called an "effects processor" that accomplishes "processing" (the modification and manipulation of sounds): one of the four functions of electronic music-making machines (along with synthesis, sampling, and sequencing) (Butler 2006, 56-57, 60). Effects processors and mixing boards contain knobs and sliders that facilitate continuous changes to musical parameters. Butler calls these knobs and sliders "continuous controllers" $(2014,71)$. They can change parameters through technologies such as filters and LFOs with physical or digital interfaces, ${ }^{(9)}$ and they can be utilized in live performance (as described in Butler 2014,70-87), or in studio production (Wright 2017, chap. 5). The physical and digital interfaces that producers work with influence the shapes of created musical gestures, and also the perception of those musical gestures by producers, fans, and analysts. ${ }^{(10)}$

[2.2] Digital interfaces often run inside of DAWs (digital audio workstations). These are software programs that are immensely important to the production of EDM. They are increasingly becoming the primary tools for popular-music creators to organize and improvise their music (D’Errico 2018; Wright 2017, 202-6). In DAWs, sound clips are represented as building blocks of music that can be changed, but are almost always quantized to the tempo and the key of the music. Discrete melodies are usually created with the "piano roll," a vertical representation of the black and white piano keys. Continuous processes can be created through "in-time" movement of virtual knobs and sliders, and can also be programmed into preset sounds that are stored in sound banks 
(such as when a particular bass sound has vibrato programmed into it), or created through automation curves.

[2.3] In music production, automation refers to changing musical parameters "automatically" rather than manually. When automation occurs, it is the technology that is controlling the changes itself, rather than human agency (Butler 2014, 223). This can help reduce the workload for DJs both in live performance and in the studio (Snoman 2009, 74-76; Wright 2017, 297). In DAWs, automation is produced with "automation curves" that represent continuous changes to musical parameters. The default shape of these is linear, but they can be altered by clicking and dragging them into any two-dimensional shape. Different shapes (such as exponential or sinusoidal curves) impact our perception of the continuous processes, as discussed in the Appendix. Example 3 shows a screenshot of the DAW file for "Beneath with Me" by deadmau5 \& Kaskade f. Skylar Grey (2016). The discrete building blocks of sound clips can clearly be seen, as can the piano roll in the bottom left, but continuous processes are also represented, since the orange lines in the middle of the picture are automation curves.

[2.4] Edward Wright (2017) discusses how automation curves in DAWs are a vital means of expression for house and techno music specifically. "While other forms of popular music use automation, it is not as directly responsible for such drastic transformations of musical material, transformations that are so significant to the construction and consumption of house and techno tracks (299-300)." In the minds of the producers he interviews, automation 1) is linked with creativity and experimentalism, 2) is a means of creating momentum, and 3) allows "the desired balance between repetition and constant change" (Wright 2017, 300).

[2.5] It is true that continuous processes are often improvised during live performance or added to EDM tracks through automation after the main melodies, harmonies, rhythms, and form have been composed, and that many continuous processes (especially the shorter ones) can be described as "effects" that are not part of the ontology of a musical work. Put another way, continuous processes are usually understood as ontic rather than ontological. In describing his compositional method, deadmau 5 talks about adding continuous processes such as "swells and dips" or "little weird effects and small variations" after the primary melodies and rhythms have been added (Zimmerman 2016, Lesson 13, 0:07 and 4:52). However, this does not diminish their importance. Many "effects" such as vibrato, siren sounds, the tremolo effect, and increasing delay or reverb, are highly salient and influence the "feel" of the music. Furthermore, in most of today's popular EDM tracks, long crescendos and pitch slides in buildup sections are highly important continuous processes that are central to the form and aesthetics. A group of continuous processes that increase tension leading towards a musical climax in this way can be called a "riser" or a "soar."(11)

[2.6] Continuous processes have been discussed systematically in analytical and compositional theories of electroacoustic music (Blackburn 2011; Callender 2004; Smalley 1997; Wishart 1996). For example, Trevor Wishart develops a system for classifying what I call continuous processes according to 1) magnitude or range (for example the depth of a pitch bend), 2) morphology/shapes, and 3) second-order morphology (based on rate of change) (Wishart 1996, 119-20). However, existing scholarship on EDM and popular-music analysis has focused on only certain types of continuous processes such as short effects or long risers, and not discussed them systematically (M. Latour 2018; Peres 2016; Solberg 2014). In this article I build on previous scholarship by highlighting many different types of continuous processes, which affect different parameters and have a variety of functions in contemporary EDM.

\section{Three General Functions of Continuous Processes}

[3.1] Continuous processes represent the uncomfortable notions of change and instability. This is why they are used to symbolize fear, volatility, mental illness, and the like in music for films and video games (Brownrigg 2003, 118-21; Larson 1996, 65). It is also why they are used most often in EDM during breakdown sections and buildup sections, when disorientation and tension are the primary aesthetic traits.

[3.2] Broadly speaking, continuous processes add variety and complexity to music that is otherwise very discrete and repetitious; in EDM, melodies and harmonies are relatively simple, if they exist at all, and rhythms, while complex and groovy, repeat in short loops incessantly. ${ }^{(12)}$ As discussed 
above, continuous processes are often understood as "effects" that are not fundamental to the identity of a "work," but only to the identity of a particular text or performance (a particular version of the work) (Butler 2014, chap. 1). In contemporary culture where remixes are copious, continuous processes are not often part of the original "stems" of a track, but instead are added near the end of the production process or improvised during live performance (Butler 2006; 2014; Zimmerman 2016). This does not mean that continuous processes are not salient or not important to the musical structure of tracks though, as the examples in this article show. Specifically, the functions of continuous processes in contemporary EDM can be described in three general categories: ornamentation, orientation, and disorientation.

\section{Ornamentation}

[3.3] Continuous processes can have various lengths, from less than a beat to many measures. This raises questions of limits. How short or long can a continuous process be before the continuous changes in it are imperceptible to the ear? How much change in a musical parameter is required for a process to be perceived as continuous? These are questions that have been discussed elsewhere, and there are borderline cases (Smith 2019, 140-51), but for now, I merely want to point out that continuous changes in musical parameters that last for a measure or less can still be important aspects of a composition. One way this is evident in contemporary EDM is when short, repeated continuous processes are used as effects to embellish discrete melodies and rhythms in a manner similar to Baroque ornamentation. The "tremolo effect" is one specific way that this is often accomplished. In music production, the tremolo effect refers to repeated continuous volume changes that create pulsating waves of sound. This is usually accomplished with an LFO.

[3.4] A good example of the tremolo effect occurs in "Imaginary Friends" by deadmau5. It is used throughout most of the introduction, but is especially noticeable from 0:53 to 1:16. Throughout this section, the harmonic progression is ornamented with rhythmic volume swells. The articulation of the notes seems to start at the peak points of the waves. The amplitude graph showing these volume waves is shown in Example 4 and the spectrogram is shown in Example 5. It is noteworthy that the waves show up clearly on the spectrogram, meaning that short filter sweeps are also being heard since the peak points of the wave have the most harmonics. The repeated changes in perceived volume are due to both the amplitude and the frequency content being altered.

[3.5] This section of the piece also utilizes longer continuous processes. Notice how in both the amplitude graph and the spectrogram, the waves gradually become taller and wider. This indicates that the section has an overall increase in volume due to both increasing amplitude and frequency content, which create brighter and more resonant sounds. The time it takes to get from the peak of the wave to the valley also increases, so that the rhythms no longer seem to be articulated almost instantaneously, and the tremolo effect is more noticeable, especially starting at 1:09.

[3.6] Another track that uses continuous processes as ornamentation is "Find a Way" by acclaimed American producer Wolfgang Gartner, which features vocals from the hip-hop artist Snow Tha Product. For example, in the song's final buildup (2:30-2:45, transcribed in Example 6), the Synth part features short pitch slides around the F\#s within each sixteenth-note figure. In the final core section (starting at 2:45, measure 9 in the example), the repeated $B^{\text {q }} \mathrm{s}$ in the vocal part are embellished with short continuous pitch slides. There are many specific ways in which short continuous processes can provide ornamentation to EDM melodies and rhythms, and they are not the focus of this article, but it is worthwhile to notice how producers utilize the affordances of electronic technologies to embellish their music and make it distinctive.

\section{Orientation}

[3.7] One of the most common functions of continuous processes is to orient the perspectives of listeners. They are often crucial in helping listeners know "where they are" in the formal or metrical structure of a track. In buildup sections, they usually increase tension leading towards the climactic goal of the "drop," when the "beat" (usually the kick drum and sometimes the bass line or low frequencies in general) is (re-)introduced into the texture after a prolonged absence (Butler 2006, 246-47; Sayre 2014; Snoman 2019, 332). In this context, the continuous processes are providing orientation to listeners through intensification. Ragnhild Solberg (2014) shows the importance of continuously rising pitch slides as an extremely common aspect of buildup sections 
in EDM tracks. She and Hawkins (2003) both incorporate spectrograms into their analyses that show the continuous nature of buildups (specifically the gradual increase in high frequencies) and the discrete, instantaneous nature of the drop. Asaf Peres (2016, 75-84) talks about "gradual ascending gestures" (filter sweeps and pitch slides) in buildup sections of contemporary pop songs, noting that the sonic syntax of "setup-buildup-peak" he describes has its origins in EDM $(2-3,73)$. Furthermore, on filter sweeps in buildup sections, Snoman says "It is these filter movements that are typically used to create tension and movement in all forms of dance music and have formed the basis of the most well-respected dance records" $(2009,226)$.

[3.8] The main aesthetic of buildup sections is tension, which orients listeners toward the upcoming drop. Continuous processes play a significant role in this, leading the music toward a specific goal point. This is because the most common and salient types of continuous processes in buildups are ascending pitch slides or noise sweeps, and crescendos through increased volume or increased number of sounds in the texture. Both ascending gestures and crescendos are metaphorically associated with growth and increasing tension (Brownrigg 2003, 118-21; Huron 2006, 326; Patty $2009,329)$, so they contribute to the sense that buildup sections are tense and moving towards a clear goal.

[3.9] Buildup sections also usually include drum parts that articulate metrical beats strongly and consistently (as opposed to breakdown sections that usually do not). Often it is just the high percussion sounds of the snare drum and hi-hat or claps on backbeats that are used, which provides metrical stability without the fully firm grounding that is saved for the upcoming core. This combination of ascending gestures and crescendos with clear and consistent beats creates tension without disorientation. This also makes buildup sections have a sense of renewed energy and excitement that engenders a sense of expectation in dancers, and allows them to prepare for the upcoming drop by re-entraining themselves to the beat. Snoman recognizes the importance of the return of the drums at the beginning of the buildup section, saying "the drum rhythm picks up again ... signifying that the track is about to come back" (2009, 226-27).

[3.10] In general, buildups are more prominent, more tense, and more often used than in previous decades, especially in genres like trance, progressive, and big room house (Butler 2006, 226; Solberg $2014,65)$. They are almost ubiquitous not only in contemporary EDM but contemporary pop music in general (Adams 2019; Osborn 2019; Peres 2016, 73-125). Perhaps because of this pervasiveness, buildup sections are also often formulaic. The first part of the formula features a general increase in energy through techniques such as crescendos, ascending pitch slides, filter sweeps, and rhythmic diminution culminating in the "snare roll." Three out of these four trademark signifiers for buildups involve continuous processes. The fourth, however, is based on clear, easily followed discrete rhythms that are in the drum parts (see Example 1).

[3.11] This general increase in energy usually lasts for about seven measures (sometimes more, sometimes less), and it is followed by the second part of the formula, a sudden drop off in energy, sometimes called a "break" (Peres 2016, 93). Not all buildups utilize this feature, but many do. Often most of the sounds are completely cut out of the texture except for one or two that serve a special function I call the "cue."(13) This is a short anacrustic motive that is usually a vocal line, a speech sample, a melodic hook, or even just a single snare drum hit. It can last one beat, two beats, a whole bar (four beats), or rarely three beats. Cues are often self-referential and humorous, making fun of their obviousness, as in the spoken line "we came to smash this club" during the track "Smash" by DJ Exodus (2014). After the sudden drop off and the cue comes the instantaneous drop, the climactic moment when all of the elements, including the drums and the bass, return to the texture at the start of the core. The intensity and thrill of this moment is for many listeners the most pleasurable part of the EDM experience (Solberg 2014; Solberg and Jensenius 2016). DJs and producers are aware of the formulaic nature of some buildups. Deadmau 5 describes the formula as uncreative and cliché in his masterclass (2016, lesson 13, 10:45-12:00).

[3.12] Continuous processes increase tension in these situations because they create a sense of expectation (Snoman 2019, 332). They also feature constant changes in sonic parameters that generate tense responses in listeners, such as ascending frequencies, crescendos, and timbral changes (Huron 2006, 326; Patty 2009, 329-39). When these techniques are used before climaxes in contemporary popular music they are often referred to collectively as a "riser" and the ascending elements are called "uplifters" (Peres 2016, 19; Solberg 2014, 70). However, continuous processes are also often used to decrease tension with decrescendos and descending gestures that are 
sometimes referred to by the oxymoronic term "downlifters" (Smith 2019, 23; Zen World 2017). Uplifters and downlifters occur right before and after hypermetric downbeats, establishing duple hypermeter at multiple levels, and orienting listeners to these structural marking points when new sounds are often introduced (Snoman 2019, 327-28). Usually, the uplifters and downlifters will be longer and louder in the mix when corresponding to more important hypermetric downbeats, as opposed to less important ones at midway points of sections. ${ }^{(14)}$

[3.13] Going back to "Find a Way" (Example 6), notice how there are multiple continuous processes leading up to the climactic drop at 2:45. At 2:15 in the track (the start of the audio for Example 6), the final breakdown section starts. The "breaking down" aspect is less obvious than in many EDM tracks, but it is signified here by the snare drum and hi-hat suddenly (that is, discretely) dropping out. This section also contains an extended continuous transformation of the timbre in Snow Tha Product's voice. It seems to become deeper and more conventionally masculine, even though the pitches stay the same. This effect is accomplished through the slowing down of the voice, so that its frequencies become lower, but adjusting the rhythm as it slows down so that it stays in tempo with the track. When a periodic sound signal is literally sped up or slowed down, its frequency content becomes higher or lower and thus the timbre is adjusted. In this context, the continuous changing of the voice functions as a means of disorientation from the norm, which is the function of breakdown sections generally.

[3.14] In the next section, the final buildup (2:30-2:45), Snow Tha Product's voice is changed in the other direction, speeding up, so that it becomes higher. Having been fragmented and reduced to only its last word of text, the voice then gradually transforms and seems to become less and less human. At first, the speeding up makes the voice sound whiny and tinny, but then the human element disappears altogether, so that by the end of the section it sounds like any other instrumental loop. The continuous speeding up of the voice is also reflected in the rhythmic changes that are shown in the transcription, with the notes in between the beats gradually disappearing. Notably, the song's lyrics are about "trying to find a way" and "struggling to find the words to say." In the breakdown section these words are said as the voice continuously transforms its timbre, and then in the buildup the voice is reduced to only one word and blends in with the instrumental track, so the voice is literally struggling to find any words to say.

[3.15] There are other long continuous processes that also orient listeners by leading them to the drop. The main instrumental line of the piece, just called the "Synth" part in the transcription, fades in and becomes stronger before fading out along with the voice part near the end. There are also multiple layers of uplifters rising in pitch throughout the section, helping achieve the intensification that is characteristic of buildup sections. Discrete processes also play a role in this intensification. At the start of the section the hi-hat returns to the texture but the kick drum drops out, as is common in buildup sections. The removal of the kick drum creates a noticeable feeling of absence that will be remedied with the upcoming drop at the climactic start of the final core. The hi-hat also undergoes discrete acceleration, fragmenting itself, even though the articulation of the rhythm also becomes continuously less clear.

[3.16] In the final measure of the buildup the "break" happens on beat three, with all the instruments dropping out after a loud clapping sound. The cue happens during beats three and four, and it brings back the vocal line in its normal, human form, acting as an anacrusis to the upcoming beat drop. The intensification processes in the buildup, and especially the break and cue, function as markers of orientation for listeners, who expect these signals to let them know when a core section of the piece is coming back.

[3.17] Another track that provides a clear example of continuous processes serving a function of orientation is "Tissot" by Bolivaro. Specifically, there are two recurring types of ascending pitch slides that help orient listeners, giving them "sonic instructions" about how to dance. These are referred to as Type A and Type B in the form chart (Example 7). ${ }^{(15)}$ Type A pitch slides ascend approximately from F4 to F5, as shown in Example 8. ${ }^{(16)}$ Their ascents are broken up by various attack points and cutaways to a lower register (which also slowly rises but has less definite pitch because it contains numerous short pitch scoops), but their linear automation curves still continuously rise, with no breaks in terms of pitch. In the graph, the lighter-colored parts of the line represent parts of the continuous process in the higher register that are not literally sounding because the lower pitch scoops are present instead. Interestingly, the total number of attack points for both the upper and lower lines create a rhythm that is a textbook example of discrete 
acceleration, halving the number of attack points in a unit from eight (one lower plus seven higher) to four (one lower plus three higher) to two (one lower plus one higher) to one (one lower). However, this example is slightly unusual in that each group begins on either beat four or beat two, rather than the customary beats one and three of a ${ }_{4}^{4}$ measure.

[3.18] Aside from the obvious timbre difference, the second type of continuous pitch ascent (Type B in Example 7) differs from the first (Type A) in numerous ways. Firstly, Type B pitch slides are half as long as Type A, occurring over roughly four measures rather than eight. Their pitch ascents are also larger, moving approximately from C4 to C6, almost two octaves, double the span of Type A. (17) Since the consonant target of $\mathrm{C}$ (the dominant scale degree in the key of $\mathrm{F}$ minor) is never reached, the aesthetic effect of these glissandos is one of incompleteness. It sounds as if the processes are slowing down towards the end and their goal will never be reached. In the graph of pitch slide Type B (Example 9), the last part of the line that reaches C6 is in a lighter color to represent the fact that C6 is never actually reached. Contrastingly, Type A slides do reach the consonant target pitch of $\mathrm{F}$, the tonic, and in fact even break the linear pattern in order to do so, as can be seen in the sudden increase in steepness at the end of the Example 8 graph. If the pitch were to keep increasing at the constant rate of change, then the last note would be just below $\mathrm{F}$, but in order to give the continuous process a sense of completeness, Bolivaro chose to have the last beat emphasize the tonic pitch, just before the start of the next section.

[3.19] Another difference is that this track's Type B pitch slides usually have somewhat unclear attack points, suggesting a rhythm of repeated eighth notes but not articulating them clearly. This means that the ascents could easily be perceived in a casual hearing as continuous lines with no breaks or points on the line. Even if the attacks are articulated more clearly, as in the occurrence during the second breakdown section (2:49-2:56), the continuous rise in pitch is easier to hear because unlike Type A slides, Type B slides do not cut away to another, lower rhythmic idea. An additional notable difference between Type A and Type B slides is that the latter crescendo then diminuendo, whereas Type A only crescendo, so their continuous changes of volume are more complex. However, both pitch ascents begin by fading in from apparent nothingness, which is why their time stamps vary according to how quickly they become apparent to my ears.

[3.20] The aforementioned differences between the two types may allude to why they were used at different times in "Tissot." They never occur at the same time, competing with each other, but each has their specific purpose. Type A slides are used as part of a formulaic riser, to build up the energy of the track in all five buildup sections, because they are accompanied by crescendos and discrete acceleration each time. Contrastingly, Type B slides function more as "effects" and usually occur during sections where the track is more stable, including both cores and once during what I have called a "pre-core" that provides an intermediate level of stability in between two buildup sections. They are probably used this way because even though the pitch is continuously ascending (a technique that is associated with instability), the rhythm (when it is clear) is steady. Furthermore, the volume crescendos and then diminuendos, as opposed to the Type A slides that only crescendo to lead towards a new section.

[3.21] In general, Type A ascents are used during transitory sections and Type B ascents are used during stable sections, but both are used once in places that contradict those expectations. Type A occurs for the final time in the outro, when it is presented for the first time with no harmonies to anchor it to the home key of F minor, and in a context that normally decreases the energy of a track rather than increases it. Type B also occurs once during the sectional type associated with the least stability in EDM, the breakdown. It is noteworthy that this occurrence in breakdown 2 is the one with sharpest timbre and clearest attack points, setting it apart in another way from its other manifestations. Overall, both types of pitch slides in this track are used to orient listeners to specific goal points. They create a constant feeling of energy and movement towards those goals, even during sections that are otherwise stable.

[3.22] A third example of continuous processes being used to orient listeners around structural marking points occurs in "Run" by Australian artist Alison Wonderland. This track is not in the genre of house or trance, but it still exhibits many formal characteristics of contemporary EDM tracks, and could be classified as an example of "pop EDM" or a popular song with "EDM form" (Osborn 2019; Peres 2016). In the second buildup section, which could also be classified as verse 2 (1:50-2:15), a long filter sweep in the sounds accompanying the vocals gradually fills in the 
frequency spectrum leading up to the drop (the start of a chorus), as shown in Example 10. Higher frequencies are continuously added. The result is a perceived continuous timbre change, especially in the synthesized chords. High-frequency percussion parts fade in, and at the end of the section a large crescendo occurs. All of this creates a strong drive to the climactic goal, intensified by a onebeat break right before the drop. These techniques help orient listeners by signifying what formal section of the piece they are in and signaling what to expect next.

[3.23] Continuous processes are used to orient listeners this way in many recent Top-40 songs too. In fact, the terms riser and drop have made their way into scholarship and common discourse about contemporary pop music, with slightly changed meanings from their original usage as described earlier in this article. In the context of pop music, the terms riser and drop (the latter of which is also sometimes called "pop-drop") now usually refer to entire formal sections. This contrasts with their original meanings in the EDM community, which were ascending pitch slides, and the instantaneous moment when the beat comes back, respectively. For example, compare the description of these terms as "sounds" in Snoman (Snoman 2019, 294) with the description of them as formal sections in the work of Alyssa Barna (2020) and Brad Osborn (2019, 160-161). In songs with "EDM form" (Osborn 2019, 160-61), the continuous processes in the riser section can lead to either a pop-drop or a "dance chorus" (Barna 2020). In either case, they orient listeners to the formal structure of the song.

[3.24] One track that uses both downlifters and uplifters in its final buildup section (3:01-3:16) is Redub's remix of "Summer Feeling" by Nafis. The spectrogram for the section is shown in Example 11, and is annotated to show different shapes of three continuous processes. The section starts with downlifters in the form of a linear descending noise sweep (a filter sweep applied to "noise," Peres 2016, 44) which makes the high frequencies fade out. This takes place over approximately three measures. In the fourth measure, uplifters start continuously rising over two octaves. These pitch slides take the shape of an exponential curve, ascending faster and faster towards the end. Finally, two measures before the end of the section and the start of the next core, a low-cut (high-pass) filter continuously removes low frequencies from the texture. This happens for seven beats. On the last beat of the section, tension is released and the low frequencies are reintroduced, not suddenly, but quickly with a linear motion. This quick release functions as a "cue" for listeners. The resulting shape of the two filter sweeps is a triangle, as shown in the annotated spectrogram. All of the continuous processes in this section (downlifters, uplifters, and the filter sweeps at the end) provide signals to listeners about falling and rising tension. They orient listeners to the formal structure of the track.

[3.25] Continuous processes (especially risers with uplifters and crescendos) can also be used to mislead listeners by only seemingly orienting them to the formal structure of the music. This sometimes occurs both in recorded tracks and in live performance. After a riser, dancers will immediately expect a climactic drop with many loud sounds, but this does not always happen. Sometimes the drop is only delayed by a measure or so, and other times it is entirely avoided for a long period of time. This is an important way in which artists create further tension by "teasing" audiences before resolution (Butler 2014, 221; Snoman 2019, 331-32).

[3.26] For example, in the Aback remix of "Everything is Bright" by Stoned Butterflies, pitch slides play an important role in the eight-measure sections of 1:30-1:45 and 3:00-3:15. In the key of C\# minor, a quiet tonic pitch enters the texture around beat six of this section (at 1:32.90, as shown in the graph of Example 12), and then slowly ascends a ninth up to a D\#. After this supertonic scale degree is reached, a fast and repetitive pitch wave is generated, which increases its depth quickly as the music leads toward the next section. The aesthetic effect of this whole process is what Austin Patty calls the "struggle scenario," when the rate of change slows down but intensification still increases (Patty 2009, 329-39). As this happens, listeners are being oriented to expect a climactic drop immediately afterwards. The first time, at 1:45, the music instead dramatically shifts to a lowenergy breakdown section. Then, after an extended buildup, the true drop and climax of the track finally happens at 3:15, after this process occurs for a second time.

[3.27] "Star Guitar" by The Chemical Brothers also features misleading continuous processes. Example 13 shows the graph for the first of several three-measure pitch slides in this track. Six other instances of this process occur at 1:55, 3:56, 4:42, 4:57, 5:42, and 5:57. It seems to have an anacrustic function throughout the track, leading up to important sectional markers, often where new elements are introduced to the texture, as is typical for a core section. Therefore it can be 
interpreted as a process that orients listeners to the formal and metrical structure of the track. The decreasing-accelerating shape creates a sense of dropping or falling faster and faster, like the acceleration of gravity, which helps the process feel anacrustic and goal oriented. However, the track is unusual in that these orienting pitch slides that would normally be ascending are instead descending. If a listener is hearing the track for the first time, the descending pitch slide might at first sound misleading and disorienting in relation to the formal structure, but might then be reinterpreted as an orienting marker in later iterations.

\section{Disorientation}

[3.28] A third common function of continuous processes in contemporary EDM is disorientation. This most commonly occurs in the formal part of the track called a breakdown, which is characterized by low sonic energy and a thin musical texture (Snoman 2019, 332). Often, some or all drum parts are absent from breakdown sections, meaning that keeping the beat becomes the responsibility of the dancers instead of the performers. In EDM, the drums (especially the kick drum) are the backbone of the music. They provide stability and articulate the beat, so when they are taken away, a sense of instability is created. In these situations syncopated rhythms are often heard without clear reference points to put them in context. Continuous processes often add to this sense of instability by making melodies or rhythms unclear or obscured. For example, if short, repeated continuous processes are particularly salient (as when wide vibrato is applied to a melody), they can distort the discrete melodies and rhythms and contribute to an aesthetic of disorientation. This aesthetic is important because it provides a break from the constant repetition in other sections. In live performance settings, disorientation also provides dancers with opportunities to physically rest, visually interact with the DJ, and perhaps "trip out" with the aid of drugs (Vitos 2017). ${ }^{(18)}$

[3.29] One example of continuous processes contributing to the disorienting aesthetic in a breakdown section is from "Superliminal" by deadmau5. This piece can best be thought of in compound meter, with a time signature of ${ }_{8}^{12}$, as shown in the transcription (Example 14). In the breakdown section (2:03-2:59), the main synth line of the piece, which articulates eighth notes (what would be triplet eighth notes in ${ }_{4}^{4}$ time) and its accompanying chords both hold over from the core, but the drums (on dotted quarter notes) and the bass (which provides a triple-meter feel with quarter notes in ${ }_{8}^{12}$, creating metrical dissonance) are notably absent. Continuous processes contribute to the disorientation caused by this breakdown section in multiple ways.

[3.30] The accompanying chords feature the tremolo effect throughout. In other words, even though it sounds like they have sustained notes changing every two measures on the downbeats (which helps provide some consistency and a sense of hypermeter), each chord swells continuously up and down in volume, creating rhythmic effects without re-articulation. The rhythm created by the tremolo effect is also not very clear. Sometimes it seems to be emphasizing each beat of the measure with its peak volume, and other times it seems to emphasize what would be "off beats" in simple meter (as in ${ }_{4}^{4}$ time). Other times it is not clear at all what rhythm the tremolo effect is supposed to create with its pulsations. Example 15 (the amplitude graph from near the end of the section, when the chords are featured alone without the accompanying rhythmic synth) shows that panning is also utilized significantly and seemingly randomly, which could be why the rhythms generated by the tremolo effect sound so unclear. ${ }^{(19)}$ The continuous swells of the tremolo effect and the panning therefore create rhythmic confusion in this breakdown section.

[3.31] Additionally, the main rhythmic synth line that articulates eighth notes undergoes a variety of changes in this section. Its gradual process of change takes place in three stages. Stage one is the first six measures of the section (2:03-2:15), when the synth is heard with a regular timbre, clear pitch, and clear rhythm, as it was in the previous sections of the track. Over the next ten measures (stage two, 2:15-2:33) numerous continuous processes are used to alter this sound layer. Its timbre is changed, becoming filled with more frequencies so that no single pitch is clearly defined. Another sound also fades in and articulates a rhythm of displaced eighth notes, halfway inbetween the others, creating a resultant rhythm of sixteenth notes. This is a technique that can be described as the "illusion of acceleration," since it seems as if the rhythm is speeding up but it is actually caused by a crescendo in a different rhythmic layer (Smith 2019, 54-56). ${ }^{\text {(20) }}$ Then it seems as if delay and echo effects are used to complicate the rhythm even further, so that not only is the 
pitch unclear but also the rhythm is muddled. Volume changes are also used on this sound layer at the same time. The result is that in the middle of this breakdown section numerous continuous processes are being used as effects to create confusion. Stage three (2:33-2:49) is just a fade out, in which the main synth line from the texture is gradually removed so that the tremolo effect in the chords is highlighted and the return of the eighth notes in the synth in the next section is made more powerful.

[3.32] After this fade out, the listener can entrain a sense of meter again because the chords are still repeating every two measures, and the tremolo effect is emphasizing pulses on every "off beat." However, this is something of a metric mirage. Though it temporarily sounds like ${ }_{4}^{4}$ time, the start of the next section clearly returns to $\frac{12}{8}$, so part of the function of the breakdown is to lull the listener into a metric group of pulses that will soon be contradicted. They are being disoriented from the true prevailing meter of the piece. Therefore, even with the discrete and repetitive chord changes providing some stability, the lack of drum parts combined with the continuous changes applied to the synth sound and the tremolo effect in the chords creates an aesthetic of disorientation in this breakdown section.

[3.33] Breakdown sections do not always contain a large number of continuous processes, but continuous processes often take on a more significant role in them than they do in cores. Along with the typical removal of drums and metrical dissonance created by syncopated layers, continuous processes contribute to the aesthetics of low energy, instability, and disorientation that define breakdown sections.

[3.34] Disorientation can also occur in intro sections, especially if they are long, in which case they often function like a breakdown, preceding a buildup. This happens in "Cthulhu Sleeps" by deadmau5, due to continuous acceleration and deceleration. In the second part of the long threepart intro (from 2:00 to 3:00), there are three different sound layers, two of which accelerate at different rates and one of which remains constant. All three were introduced in the previous section, and were initially repeated at constant (isochronous) intervals.

[3.35] Example 16 shows the speed of each of the three layers, in attacks per measure, for the thirtytwo measure section that features continuous acceleration and deceleration (2:00-3:00). This is an imperfect method of measurement, in that the graph looks jagged and not perfectly continuous, especially in the pitched synth. However, this was the most practical method for my purposes. Only deadmau5 knows how he created these accelerating effects, whether it was through turning real or virtual knobs or entering values in a precise table in a software program. But to the listeners the actual acceleration and deceleration occurs at a precise enough level that it sounds smooth and continuous. ${ }^{(21)}$

[3.36] Two of the three rhythmic layers begin the section by accelerating. The other rhythmic layer maintains isochronous beats, so the tempo of the track remains static, linking this section to the surrounding ones that are more stable rhythmically. Of the two layers that accelerate, one does so much more rapidly, to the point that it almost merges into one continuous sound with no perceptible subdivisions. This sound layer is the most salient and recognizable feature of the track as a whole, and it can be thought of as representing the mythical Cthulhu monster. ${ }^{(22)}$ I will call it the "rhythmic synth" for lack of a better term, because unlike the other two layers it does not have an easily identifiable pitch.

[3.37] The behavior of the rhythmic synth in this section can roughly be mapped into three phases. First is the acceleration phase that takes place in measures 1-13 of this thirty-two measure section. During this section it can be imagined that the Cthulhu is waking up. Notice how the rate of change is fairly constant but towards the end it accelerates quite quickly. The second phase is when the rhythmic synth reaches its peak speed in mm. 14-17. This is when it almost blurs into one continuous sound, and the acceleration is most easily perceived as continuous not discrete. The third phase is the deceleration phase in mm. 18-32, when the rhythmic synth slows down to even slower than it was before the changes started. At this point the Cthulhu can be imagined to be going back to sleep, settling into a calmer state.

[3.38] The second layer has an obvious element of pitch to it, so I will name it the "pitched synth." This sound was introduced in the previous section of the piece, fading in around 1:45 and having a steady rhythm, albeit a metrically dissonant one with the beat. ${ }^{(23)}$ The pitched synth accelerates 
like the rhythmic synth, but it does so at a much slower rate, and also continuously raises its pitch at the same time from approximately F4 to D5. ${ }^{(24)}$ Unlike the rhythmic synth, the pitched synth only accelerates until it fades out around 2:47; it never decelerates. The use of only acceleration, but at a slow rate, marks this layer as different from the other two in the texture. The third sound layer, the "pulsing drone," sounds like an alarm clock repeatedly going off. It maintains the tempo of the track while the pitched synth and rhythmic synth accelerate and/or decelerate at significantly different rates. All three of these layers put together creates a highly disorienting aesthetic.

[3.39] It is also interesting to track the volume and perceived salience/prominence of each of the three layers in this section. This is represented by the thickness of the lines in Example 16. The rhythmic synth stays strong throughout, but at times one or both of the other layers seem to be competing with it for preeminence. The pulsing drone starts the section with a strong, harsh sound and accentuated overtones, but then it mellows and continuously changes its timbre as it fades to the background while the two accelerating layers reach their peak speeds. ${ }^{(25)}$ Then as the pitched synth fades out, the pulsing drone gets louder, highlighting the absurd slowness of the rhythmic synth and preparing the listener for the upcoming section when the pulsing drone will take over and repetitive discrete elements will be much more salient. Overall, the section begins and ends with both discrete and continuous processes, but in the middle the continuous processes are most salient and brought to the foreground. ${ }^{(26)}$ This section of "Cthulhu Sleeps" provides a good example of how continuous processes can function as a way of disorienting listeners.

\section{One Summative Example}

[4.1] "Be Strong" (The Loops of Fury Mix) by Elite Force features all the functions of continuous processes discussed throughout section 3 , and it is a good summative example for this article. It shows how continuous processes with various functions of ornamentation, orientation, and disorientation interact over the course of an entire piece. A form diagram of the piece is shown in Example 17. ${ }^{(27)}$

[4.2] In this track, a multitude of short pitch slides are used throughout, including in the intro and main core sections. They function as ornamentation for repetitive rhythmic patterns. From the very beginning of the track the main melodic loop that lasts one measure is heard repeatedly. As shown in the transcription (Example 18), the downbeat is clearly articulated by a stable bass note, but the other beats contain many short ascending pitch slides (scoops). In addition to the scoops between each pair of sixteenth notes, the starting and ending points of each short scoop continuously get higher. Thus, there are two scale levels of pitch slides going on here: one that takes place repeatedly over half a beat, and one that takes place over three beats. Both of these scoops are ornamentations of the main melodic figure that loops in most of the track, including all the core sections.

[4.3] In the first buildup section (0:45-1:00), repeated, multidirectional waves of pitch motion occur. Each ascending and descending cycle of the wave can be thought of as one short continuous process. As was the case in "Imaginary Friends" (Examples 4 and 5), the shorter processes are embedded within a longer one, since the short pitch waves transform over the course of the buildup section. Firstly, the speed of the wave cycles increases through acceleration, and secondly, the pitches at the high and low points of the wave continuously ascend. The continuous pitch ascents at two different levels of length (such that shorter scoops are embedded within a longer ascent), mimic the processes in the main loop transcribed in Example 18. The buildup section features an expanded version of this technique. The continuous acceleration, combined with the ascending frequencies (including an ascending noise sweep), orients listeners by signaling that this section is a buildup. After the one-beat cue of "woo!" the first core section begins.

[4.4] The breakdown section (2:45-3:15) is sixteen measures long, broken up into two eightmeasure parts. It is preceded by an ascending noise sweep and starts with a descending noise sweep. These are uplifters and downlifters that orient listeners by highlighting the sectional boundary. In the first part of the breakdown (2:45-3:00) only two rhythmic layers are present. One melodic motive from the core holds over. This repeated vocal hook ("be strong") is the hook of the song, which comes in during the first of every four measures and fades out continuously over the next three. ${ }^{(28)}$ This is yet another continuous process that contributes to the instability valued in breakdown sections. 
[4.5] The second rhythmic layer in the section is more perceptually salient. It utilizes repeated filter sweeps that add or remove high frequencies in continuous waves. The shape of the waves seems to indicate exponential growth for the ascending part and exponential decay for the descending part, as shown in the spectrogram (Example 19). The filter sweeps continuously change the timbre and the volume of the sounds, making up-anddown swells, with the peak of each wave generating a rhythmic pulsation. This creates mostly clear rhythms that are metrically dissonant with the non-sounding but still-internalized beat. ${ }^{(29)}$ But since the drums are not present for eight whole measures (2:45-3:00), the beat is easy to lose, and the continuous processes contribute to a sense of disorientation. When the drums come back in at 3:00 for the second half of the breakdown, the meter of the track is re-clarified. However, the continuous filter sweeps are still prominent and now create explicit metrical dissonance with the sounding reference points of the drums.

[4.6] After this, the final buildup happens (3:15-3:45). It is fairly formulaic, orienting listeners to their place in the formal structure. In this sixteen-measure section, the drums follow the standard EDM rhythmic diminution/discrete acceleration pattern by playing half notes in the first eight measures, quarter notes in the next four, eighth notes in the next two, then sixteenth notes for the last seven beats before the cue. This is the standard way of accomplishing a snare roll at the standard pace, although here it is accomplished with a kick-drum sound.

[4.7] Another way in which the tension increases in this formulaic buildup section is through the very loud pitch slide that occurs for almost the entire sixteen-measure section. This pitch slide is even more salient than usual ones because the glissando is applied to a chord, not just an individual pitch. Towards the end of the process, a noise sweep joins it, creating an even stronger drive towards the goal point. This can be seen in the spectrogram (Example 20).

[4.8] A different sound layer in this buildup section features shorter continuous processes, specifically three pitch slides in every measure. This layer is relatively quiet, but it is reminiscent of the short pitch slides in the first buildup, which were one of the primary stimulants of tension in that section (0:45-1:00).

[4.9] Also during the final buildup section (3:15-3:45), the vocal hook "be strong" that was discussed in the breakdown section is still present, but now not fading out and only looping every two measures instead of four. It cuts out of the texture four measures before the drop, which creates more tension and allows listeners to focus more on the continuous processes of growth. After fifteen measures and three beats of salient continuous processes in this buildup section, there is a one-beat break that features the vocal cue of just an excited "woo," signaling the upcoming beat drop and the start of the final core in the track, when the tension is released and climax is achieved. As this section has shown, throughout this remix of "Be Strong," continuous processes serve as sonic instructions with functions of ornamentation, orientation, or disorientation.

\section{Conclusion}

[5.1] Continuous processes move between two discrete points with constant change. They are by definition unstable. Listening to them is potentially uncomfortable because our brains and bodies do not know when the change is going to stop. We can make predictions about where they will lead based on previous patterns and our expectations, but it is not until the change stops and our predictions are fulfilled at the moment we expect (or slightly after we expect) that a climactic moment of release happens (Huron 2006, 322-26). This explains why even though continuous processes function in various ways in EDM tracks, they play their most significant roles in sections that are marked by transition and change, such as intros, outros, and especially breakdown and buildup sections.

[5.2] When experiencing the emotional waves that fans and producers describe in EDM, continuous processes guide them through the structure of the music by providing sonic instructions for how to dance. Short processes that function as ornamentation often occur in core sections, and they help listeners interpret rhythms and melodies so they can dance to them with appropriate timing. Breakdown sections often feature disorienting continuous processes that are hard to interpret within the normal meter and tempo on the dance floor, which communicates to dancers that they can take a break. In buildup sections (and sometimes in other places), continuous processes such as 
uplifters and downlifters orient listeners by leading them to and from structural markers so they can coordinate their bodies to the beat again. Even though the continuous processes in buildup sections have musical tension, it is caused by a sense of expectation, such that the continuous processes orient listeners and they know what to expect. Continuous processes are a crucial part of how contemporary EDM is experienced, and they contribute to the alternation of tension and release that fans and producers of this music are referring to when they speak about riding emotional waves.

[5.3] Dancers in a club or at an EDM festival often speak about moments of ecstasy when they are intimately connected with strangers, experiencing "communion" together (Garcia 2011, 167-74; Redfield and Thouin-Savard 2017). In both club settings and workout sessions, people talk about the music moving them "up and down" and giving them a boost of energy at specific points (DeNora 2000, 89-102; Solberg 2014; Solberg and Jensenius 2016). These situations happen when listening to EDM because of the intense climaxes experienced and continuous processes that precede them. The joys of stability, of being firmly grounded, of being mentally and physically synchronized with the beat and with other bodies around you, are amplified after moments of disorientation, tension and instability, which are usually caused by continuous processes.

[5.4] This article has discussed the musical and aesthetic functions of continuous processes in contemporary EDM and provided some visual models of them to help model our listening experiences. The visual diagrams provide a heuristic for understanding the general shapes of musical gestures based on their rate of change. For future analysis, though, it is important to raise the question of how detailed our analytical methods should get in modeling change. There is a perceptual limit on how much we can hear and understand changes in sounds. ${ }^{(30)}$ Visual archetypes such as the ones in Example 2 and the Appendix provide no detail about specific musical processes, but the actual curves representing specific processes in DAWs or spectrograms sometimes provide too much detail for our own aural processing. The graphs in Examples 8-9, 1213 , and 16 are attempts at visual models with a sufficient amount of precision to map the listening experience.

[5.5] It is also important to keep in mind, though, that there are many different ways of experiencing EDM, including as a DJ, a producer, a dancer, and/or an analyst (Butler 2006, 16). These are not mutually exclusive. EDM is also heard in many different social contexts today, not just in clubs and festivals but in malls and at weddings, through car speakers and headphones. In fact, the term EDM is increasingly used less as an umbrella term for all electronic dance music and more as a specific term for mainstream, "radio-friendly" dance music based on the genres of trance and progressive house (Jóri 2021, 31-34; Wright 2017, 25-26). Regardless of our individual perspectives and perceptions, though, the shapes of continuous processes are tied up with their functions, since they represent how sonic instructions are given to listeners and dancers and the degree to which orientation, disorientation, ornamentation, and intensification are communicated.

[5.6] Future analytical research on EDM or other popular music can use the methods in this article to help answer questions of genre differentiation and the semiotic possibilities of continuous processes in different genres. For example, how often are risers used in different genres, and with what different shapes? How has this changed in EDM as a whole in the last several decades? ${ }^{(31)}$ The techniques and processes that are noticeable and valorized in specific works also depend on genre (Wiltsher 2016, 418). One specific example is the "wobble bass" that dubstep is known for (D'Errico 2015; Osborn 2018). In the study of popular music more broadly, the methods in this article could be used to discuss continuous processes in non-EDM contexts, for example vocal inflections and guitar effects in pop and rock music. The methods used here also could be applied to electroacoustic music, contributing a new way of categorizing continuous processes by their perceived general shapes and functions that adds to existing analytical scholarship of continuous processes in that repertoire (Callender 2004; Wishart 1996).

[5.7] Continuous processes contribute significantly to the musical structure and style of contemporary popular music. This repertoire that is cherished by so many deserves to be studied in a multitude of different ways, and by drawing attention to the roles of continuous processes, this article has shown how they are significant in contemporary EDM. They are an important part of how EDM is created and understood, both by creators and fans, and they contribute significantly to the joy that is created through riding the emotional waves in this music. 
Jeremy W. Smith

University of Louisville

School of Music

105 E Brandeis Ave

Louisville, KY 40208

jeremy.smith.6@louisville.edu

\section{Works Cited}

Adams, Kyle. 2019. “Musical Texture and Formal Instability in Post-Millennial Popular Music: Two Case Studies." Intégral 33: 33-45.

Barna, Alyssa. 2020. “The Dance Chorus in Recent Top-40 Music.” SMT-V 6 (4). https://doi.org/DOI: http://doi.org/10.30535/smtv.6.4.

Blackburn, Manuella. 2011. “The Visual Sound-Shapes of Spectromorphology: An Illustrative Guide to Composition." Organised Sound 16 (1): 5-13. https://doi.org/10.1017/S1355771810000385.

Brøvig-Hanssen, Ragnhild, and Anne Danielsen. 2016. Digital Signatures: The Impact of Digitization on Popular Music Sound. MIT Press. https://doi.org/10.7551/mitpress/10192.001.0001.

Brownrigg, Mark. 2003. “Film Music and Film Genre.” PhD diss., University of Stirling.

Burns, Gary. 1987. “A Typology of 'Hooks' in Popular Records.” Popular Music 6 (1): 1-20. https://doi.org/10.1017/S0261143000006577.

Butler, Mark J. 2001. “Turning the Beat Around: Reinterpretation, Metrical Dissonance, and Asymmetry in Electronic Dance Music." Music Theory Online 7 (6). https://mtosmt.org/issues/mto.01.7.6/mto.01.7.6.butler.html.

2006. Unlocking the Groove: Rhythm, Meter, and Musical Design in Electronic Dance Music. Indiana University Press.

2014. Playing with Something That Runs: Technology, Improvisation, and Composition in DJ and Laptop Performance. Oxford University Press. https://doi.org/10.1093/acprof:oso/9780195393613.001.0001.

Callender, Clifton. 2004. “Continuous Transformations.” Music Theory Online 10 (3). https://mtosmt.org/issues/mto.04.10.3/mto.04.10.3.callender.pdf.

Cohn, Richard. 1992. “Transpositional Combination of Beat-Class Sets in Steve Reich's Phase-Shifting Music." Perspectives of New Music 30 (2): 146-77. https://doi.org/10.2307/3090631.

Danielsen, Anne, ed. 2010. Musical Rhythm in the Age of Digital Reproduction. Ashgate.

De Souza, Jonathan. 2017. Music at Hand: Instruments, Bodies, and Cognition. Oxford University Press. https://doi.org/10.1093/acprof:oso/9780190271114.001.0001.

DeNora, Tia. 2000. Music in Everyday Life. Cambridge University Press. https://doi.org/10.1017/CBO9780511489433.

D’Errico, Mike. 2015. “Electronic Dance Music in the Dubstep Era.” Oxford Handbooks Online. https://doi.org/10.1093/oxfordhb/9780199935321.013.74.

- 2018. "Technologies of Play in Hip-Hop and Electronic Dance Music Production and Performance." In Critical Approaches to the Production of Music and Sound, ed. Samantha Bennett and Eliot Bates, 138-56. Bloomsbury. https://doi.org/10.5040/9781501332074.0016.

Dolan, Emily. 2012. “Toward a Musicology of Interfaces.” Keyboard Perspectives 5: 1-12.

Fink, Robert. 2005. Repeating Ourselves: American Minimal Music as Cultural Practice. University of California Press. https://doi.org/10.1525/9780520938946. 
Garcia, Luis-Manuel. 2005. "On and On: Repetition as Process and Pleasure in Electronic Dance Music." Music Theory Online 11 (4).

http://www.mtosmt.org/issues/mto.05.11.4/mto.05.11.4.garcia.html.

2011. "'Can You Feel It, Too?': Intimacy and Affect at Electronic Dance Music Events in Paris, Chicago, and Berlin." PhD diss., University of Chicago.

t' Hart, Johan, René Collier, and Antonie Cohen. 1990. A Perceptual Study of Intonation: An Experimental-Phonetic Approach to Speech Melody. Cambridge University Press. https://doi.org/10.1017/СBO9780511627743.

Hawkins, Stan. 2003. "Feel the Beat Come Down: House Music as Rhetoric." In Analyzing Popular Music, ed. Allan F. Moore, 80-102. Cambridge University Press. https://doi.org/10.1017/CBO9780511482014.005.

Holmes, Thom. 2016. Electronic and Experimental Music: Technology, Music, and Culture. 5th ed. Routledge. https://doi.org/10.4324/9781315684796.

Huron, David. 2006. Sweet Anticipation: Music and the Psychology of Expectation. MIT Press. https://doi.org/10.7551/mitpress/6575.001.0001.

In The Mix. 2018. “How To Use Automation Clips - FL Studio 20 Essentials.” YouTube video, 7:23. https://www.youtube.com/watch?v=_FhYmrp4Too.

James, Robin. 2015. Resilience \& Melancholy: Pop Music, Feminism, Neoliberalism. John Hunt Publishing.

Jóri, Anita. 2021. “The Meanings of 'Electronic Dance Music' and 'EDM'." In The Evolution of Electronic Dance Music, ed. Ewa Mazierska, Tony Rigg, and Les Gillon, 25-40. Bloomsbury.

Kronengold, Charles. 2005. “Accidents, Hooks and Theory." Popular Music 24 (3): 381-97. https://doi.org/10.1017/s0261143005000589.

Larson, Randall D. 1996. Music from the House of Hammer: Music in the Hammer Horror Films, 19501980. Scarecrow Press.

Latour, Bruno. 1987. Science in Action: How to Follow Scientists and Engineers through Society. Harvard University Press.

Latour, Melinda. 2018. “Santana and the Metaphysics of Tone: Feedback Loops, Volume Knobs, and the Quest for Transcendence." In The Relentless Pursuit of Tone: Timbre in Popular Music, ed. Robert Fink, Melinda Latour, and Zachary Wallmark, 212-32. Oxford University Press.

Law, John. 1987. “Technology and Heterogenous Engineering: The Case of Portuguese Expansion.” In The Social Construction of Technological Systems: New Directions in the Sociology and History of Technology, ed. Wiebe E. Bijker, Thomas Parke Hughes, and Trevor J. Pinch. MIT Press.

London, Justin. 2012. Hearing in Time: Psychological Aspects of Musical Meter. 2nd ed. Oxford University Press. https://doi.org/10.1093/acprof:oso/9780199744374.001.0001.

Lovecraft, H.P.. [1928] 2016. The Call of Cthulhu. The Floating Press.

Moseley, Roger. 2016. Keys to Play: Music as a Ludic Medium from Apollo to Nintendo. University of California Press. https://doi.org/10.1525/9780520965096.

Osborn, Brad. 2019. "Risers, Drops and a Fourteen-Foot Cube: A Transmedia Analysis of Emil Nava, Calvin Harris, and Rihanna's 'This Is What You Came For.'” In Transmedia Directors: Artistry, Industry and New Audiovisual Aesthetics, ed. Carol Vernallis, Holly Rogers, and Lisa Perrott, 159-68. Bloomsbury. https://doi.org/10.5040/9781501339295.0020.

Osborn, Matthew J. 2018. “Aesthetics of Rhetorics during Timbre." Enculturation 26 (May). http://enculturation.net/aesthetics-of-rhetorics.

Patel, Aniruddh D. 2008. Music, Language, and the Brain. Oxford University Press. 
Patty, Austin T. 2009. "Pacing Scenarios: How Harmonic Rhythm and Melodic Pacing Influence Our Experience of Musical Climax." Music Theory Spectrum 31 (2): 325-67.

https://doi.org/10.1525/mts.2009.31.2.325.

Peres, Asaf. 2016. “The Sonic Dimension as Dramatic Driver in 21st-Century Pop Music.” PhD diss., University of Michigan.

Redfield, Audrey, and Marie I. Thouin-Savard. 2017. “Electronic Dance Music Events as Modern-Day Ritual." International Journal of Transpersonal Studies 36 (1): 51-66. https://doi.org/10.24972/ijts.2017.36.1.52.

Reich, Steve. 2002. Writings on Music, 1965-2000. Edited by Paul Hillier. Oxford University Press.

Rothfarb, Lee. 2002. "Energetics." In The Cambridge History of Western Music Theory, ed. Thomas Christensen, 927-55. Cambridge University Press. https://doi.org/10.1017/CHOL9780521623711.032.

Sayre, Wilson. 2014. "Waiting For The Drop: The Anatomy of An EDM Song." Produced by WLRN. Streaming audio with transcription, March 27, 2014. http://www.wlrn.org/post/waiting-dropanatomy-edm-song.

Smalley, Denis. 1997. "Spectromorphology: Explaining Sound-Shapes." Organized Sound 2 (2): 107-26. https://doi.org/10.1017/S1355771897009059.

Smith, Jeremy W. 2019. "The Salience, Shapes, and Functions of Continuous Processes in Contemporary Electronic Dance Music." PhD diss., University of Minnesota.

Snoman, Rick. 2009. Dance Music Manual. 2nd ed. Focal Press.

2019. Dance Music Manual. 4th ed. Routledge.

Solberg, Ragnhild. 2014. “'Waiting for the Bass to Drop': Correlations between Intense Emotional Experiences and Production Techniques in Build-Up and Drop Sections of Electronic Dance Music." Dancecult: Journal of Electronic Dance Music Culture 6 (1): 61-82. https://doi.org/10.12801/1947-5403.2014.06.01.04.

Solberg, Ragnhild, and Alexander Refsum Jensenius. 2016. "Pleasurable and Intersubjectively Embodied Experiences of Electronic Dance Music." Empirical Musicology Review 11 (3-4): 301-18. https://doi.org/10.18061/emr.v11i3-4.5023.

Traut, Don. 2005. “'Simply Irresistible': Recurring Accent Patterns as Hooks in Mainstream 1980s Music." Popular Music. 24 (1): 57-77. https://doi.org/10.1017/S0261143004000303.

van Buuren, Armin. 2018. “MasterClass I Armin van Buuren Teaches Dance Music.” Streaming video. https://www.masterclass.com/classes/armin-van-buuren-teaches-dance-music.

Vitos, Botond. 2017. “The Dance Floor Experiment: Researching the Mediating Technologies and Embodied Experiences of Electronic Dance Music Events." Popular Music and Society 40 (2): 131-50. https://doi.org/10.1080/03007766.2015.1094903.

Wiltsher, Nick. 2016. "The Aesthetics of Electronic Dance Music, Part I: History, Genre, Scenes, Identity, Blackness." Philosophy Compass 11 (8): 415-25. https://doi.org/10.1111/phc3.12333.

Wishart, Trevor. 1996. On Sonic Art. New and rev. ed. Edited by Simon Emmerson. Harwood.

Wright, Edward. 2017. "Making Hammers With Art: The Producer of House and Techno.” PhD diss., University of Toronto.

Zen World. 2017. “The Art of The EDM Build Up.” Website tutorial, July 9, 2017. https://www.evosounds.com/single-post/2017/07/09/The-Art-Of-The-EDM-Build-Up.

Zimmerman, Joel. 2016. "deadmau5 Teaches Electronic Music Production." Streaming video. https://www.masterclass.com/classes/deadmau5-teaches-electronic-music-production.

\section{Discography}


Alison Wonderland. 2015. “Run (Official Video).” EMI Recorded Music Australia. YouTube. https://www.youtube.com/watch?v=Qc-aNL6egpY.

Bolivaro. 2013. “Tissot (Original Mix)." Tissot. Pyro Records PYO33. MP3.

The Chemical Brothers. 2008. “Star Guitar.” Brotherhood. Virgin Records 05099923419650. CD.

Deadmau5. 2010. "Cthulhu Sleeps." 4x4=12. Ultra Records UL 2518-2. CD.

. 2012. "Superliminal." > Album Title Goes Here<. Ultra Records UL 7841-2. Mp3.
—. 2016. "Imaginary Friends." W:/2016Album/. Mau5trap Recordings MAU5CD023. MP3.

Elite Force. 2011. “Be Strong (The Loops of Fury Mix).” Be Strong. U\&A Recordings UAA057. MP3.

Exodus. 2014. “Smash.” Freakshow Vol. 1. Peak Hour Music PHMC02. MP3.

Nafis. 2013. "Summer Feeling (Redub's at 5am Remix)." Summer Feeling. Progressive House Worldwide PHW091. MP3.

Stoned Butterflies. 2012. "Everything is Bright (Aback Remix)." Everything is Bright EP. Baci Recordings BR0026. MP3.

Wolfgang Gartner, feat. Snow Tha Product. 2017. "Find a Way." Armada Music. YouTube. https://www.youtube.com/watch?v=w_73L8GrZ04.

\section{Footnotes}

1. “MasterClass | Armin van Buuren Teaches Dance Music" (van Buuren 2018, lesson 17, 0:00$0: 30)$.

Return to text

2. Long before EDM existed, Ernst Kurth theorized about "force waves" in music, which have "escalatory and de-escalatory undulations that shape the musical flow" (Rothfarb 2002, 943).

Return to text

3. Butler describes the importance of heterogeneous, stratified textures in EDM generally (2006, 93, 182, 224).

Return to text

4. I am using the word "processes" to describe these phenomena rather than "motions" because it describes both continuous and discrete techniques better as an umbrella term. However, "motions," "gestures," or "changes" are also useful words for the techniques I describe, especially continuous ones, and therefore I also use them occasionally.

Return to text

5. The most common way of altering a sound's timbre is by manipulating the amplitudes for each of its ADSR (attack, decay, sustain, release) components. The most common way of changing the frequency content of sounds is by applying filters (Holmes 2016, 226-29). Filter sweeps do change timbre, but they also change volume and pitch, so I place them in a separate category.

Return to text

6. Another important aspect of EDM that has been discussed in this journal and elsewhere is repetitiveness and continuous flow both within and between tracks (Butler 2014, chap. 4; Fink 2005, chap. 1; Garcia 2005). However, this is not the kind of continuity discussed in this article. Return to text

7. For a summary of scholarship on EDM genres see Wiltsher (2016, 416-19).

Return to text

8. For further definitions of these terms and their applications in EDM see Butler (2014, chap. 1).

Return to text 
9. LFOs (low frequency oscillators) are generally used to create continuous alterations of pitch (vibrato) or amplitude/volume (the tremolo effect), but they can be applied to modulate any parameter in the wave shape of a low, inaudible frequency.

Return to text

10. For more on how interfaces influence the creation and perception of music see D'Errico 2018, De Souza 2017, Dolan 2012, B. Latour 1987, Law 1987, and Moseley 2016.

Return to text

11. The term "riser" does not have a universally accepted meaning. In general it refers to any ascending pitch slide, but sometimes it is used in this more specific way to refer to a group of continuous processes including ascending pitch slides, filter sweeps, and crescendos, or the formal section in which they are used, just before a climax (Barna 2020; Osborn 2019, 160-61; Peres 2016, 19). For information about the "soar" in popular music, see James (2015).

Return to text

12. The prominence of clear melodies and harmonies is one of the important markers of distinction between various EDM genres. Trance and house are much more melodic, whereas techno and "drum and bass" are more focused on rhythms and drum parts.

Return to text

13. Peres hints towards this feature, saying that sometimes the vocals are left by themselves during the break, but he does not give it a name (Peres 2016, 93).

Return to text

14. Peres shows that this is true in contemporary Top-40 music as well, since he discusses numerous examples of uplifters (including both ascending noise sweeps and pitch slides) occurring just before the midpoint of choruses in popular songs (Peres 2016, 79-82).

Return to text

15. For explanations of the sectional types in EDM tracks see Butler (2006, 221-24).

Return to text

16. I created this graph and others like it in the article through my own close listening, also aided by tools in audio software programs. For measuring pitch slides I use the scale of semitones (rather than frequency in hertz) since it is the scale that most easily and accurately reflects musical perceptions of distance (at least for Western listeners). It does reinforce discrete thinking, but the graphs would look quite different and be more difficult to follow if they had frequency in hertz along the $y$ axis.

Return to text

17. The linear motion is harder to hear in Type B because it also fades in, so the first couple of notes are basically inaudible. Yet when one assumes that the pitch slide starts on C4 at the beginning (even though it is inaudible) and moves up 1.5 semitones every beat (a much higher rate of change than 0.375 for Type A), the pitches line up so that after sixteen beats the next pitch (on beat seventeen, which never comes) is C6.

Return to text

18. In the self-referential track "This is the Hook" by BSOD, the lyrics describing the second breakdown say "Now you should notice the dancefloor is reacting. Look up from your decks. Look at the audience. It is important to show that you are alive."

Return to text

19. Panning is the change in volume between left and right speakers in stereo audio.

Return to text

20. The "illusion of acceleration" effect is reminiscent of phasing techniques in the minimalist works of Steve Reich, who called it "the process of rhythmic construction" $(2002,76)$ and "the process of substituting beats for rests" (2002, 73). Richard Cohn (1992) also referred to this effect in Reich's music as "transpositional combination of beat-class sets."

Return to text 
21. Even though the perceived effect is one of acceleration and deceleration, the speed changes were probably accomplished with the continuous turning of an LFO knob clockwise and counterclockwise to alter the rate of waves being produced.

Return to text

22. The Cthulhu monster originated in H.P. Lovecraft's short story "The Call of Cthulhu" ([1928] 2016).

Return to text

23. The repeated rhythmic value here is roughly equivalent to a double-dotted eighth note, a.k.a. seven thirty-second notes or $7 / 8$ of one beat.

Return to text

24. At a more precise level, it sounds like there are multiple layers to what I call the "pitched synth," only one of which is pitched and the other one is rhythmic, but I group them together because they are easily perceived as one unit.

Return to text

25. Specifically, by mm. 7-8 of the section the low frequencies are emphasized much more than at the beginning.

Return to text

26. For most of the rest of the piece, the rhythmic synth is only heard on the off beats as one of many sound layers in the cores, and the pitched synth is heard accelerating again at the start of the second core (which is unusual, in that continuous processes such as acceleration rarely occur in cores).

Return to text

27. There are several subsections within Core 1 that would be interesting to study in future research. Two subsections that blur the boundaries with characteristics of multiple sectional types are 1:45-1:59 and 2:30-2:44.

Return to text

28. For literature on "hooks" in popular music see (Burns 1987; Kronengold 2005; Traut 2005). Return to text

29. In a situation like this, the beat from the rest of the track is maintained internally as a "virtual reference structure." (Danielsen 2010,4).

Return to text

30. For a discussion of these perceptual limits for pitch, rhythm, volume, and timbre see BrøvigHanssen and Danielsen 2016; Danielsen 2010; t' Hart, Johan, and Cohen 1990 , 32; London 2012, 27 29; Patel 2008, 86.

Return to text

31. These questions have been hinted at already in some scholarship, but a more thorough discussion would be highly useful. (Butler 2006, 226; Solberg 2014, 65).

Return to text

32. As shown in this video (around 2:35), the preset shapes in FL Studio (another DAW) include "smooth," "single curve" (which can be manipulated to become various types of exponential curves), "double curve" (which can be like a cubic function or a sigmoidal S-curve), "stairs," "smooth stairs," "pulse," "wave" (which can create sinusoidal shapes), and "half sine." https://www.youtube.com/watch?v=_FhYmrp4Too (In The Mix 2018). Interestingly, two of the other most popular DAWs (Ableton Live 10 and Logic Pro X) do not currently have this feature, although it can be replicated by "automating the automation" through LFOs with different wave shapes, and there are various plug-ins available with similar features.

Return to text

33. Sometimes continuous processes can be modeled well with sigmoidal curves (S-curves), which almost plateau at their beginnings and endings, having a high rate of change in the middle (Smith 2019, 219-21, 227-28, 304-6). However, none of the examples in this article, which best demonstrate 
the functions of continuous processes that I want to show, have this shape.

Return to text

34. Technically, according to scientific definitions, all four of these graphs are "accelerating" because they are changing their velocity. However, I find it more useful to describe those graphs with increasing rate of change as accelerating and those with decreasing rate of change as decelerating.

Return to text

\section{Appendix: The Shapes of Continuous Processes}

[A.1] Continuous processes can be visually represented by lines and curves in two-dimensional space, where time is on the $x$ axis and another parameter such as frequency or tempo is on the $y$ axis. Many continuous processes are created with these representations in DAWs, using automation curves or LFO tools. The parameter changed with these technologies can be anything the DAW can manipulate, leading to a huge variety of possibilities. Of course a composer could add many different automation curves to one sound layer or many sound layers.

[A.2] Automation curves can be in any shape, including preset ones or ones created by manually dragging the curve around with the mouse. ${ }^{(32)}$ They can create changes for any sound channel(s), and for any parameter(s) or even threshold limits. The shapes that are created, especially for continuous processes that are prominent in the track, significantly influence the aesthetic impact of the music. Most continuous processes used in EDM behave in at least some sense like linear, sinusoidal, or exponential functions, as the examples in this article show. Basic graphs of these functions and their derivatives are in Examples 21-23. The derivative functions are important because they represent the rate of change. We can never know all the specific types of curves used since they can be customized. However, based on their rate of change their shapes can be discussed in general terms, using the shapes in Examples 21-23 (and others as necessary) as reference points.

(33) The heuristic methods used in this article help us understand our perception of these musical gestures as listeners and dancers, but they are also rooted in a poietic perspective, since the shapes are usually visible and precisely controlled in DAWs.

[A.3] Many of the general shapes used in EDM can be easily represented by one of the four basic types of exponential functions (based on horizontal and vertical flipping of the simplest exponential function). This is shown in Example 23. ${ }^{(34)}$ Although each of these shapes could also be approximated with logarithmic functions, rational functions, or even radical functions, I am choosing to think about the shapes as varieties of exponential functions because they contain horizontal asymptotes and not vertical asymptotes. An asymptote is a line that is approached more and more closely but never touched by a curve. In musical perception, horizontal asymptotes make sense as models of our listening experiences, since it often sounds like volume or pitch is approaching a plateau or a threshold, when the differences in the amount of change are becoming less and less noticeable. A vertical asymptote would not make sense in musical perception, since the $x$ axis represents time, and time cannot be stopped.

[A.4] As shown in the graphs of Examples 21-23, linear functions have a constant rate of change, and exponential functions increase or decrease their rate of change over time (in magnitude, a.k.a. absolute value). Sinusoidal functions have their rate of change sometimes increasing and sometimes decreasing. Specifically, it increases in between peaks and valleys, and decreases as the peaks and valleys are approached. Some of the continuous processes I discuss in EDM pieces can be mapped onto common mathematical formulae more precisely than others, but even if they are not a perfect match, applying different shapes to them gives us a better idea of how the continuous processes that influence our hearing and our dancing work, and why composers may choose specific rates of change over others for different parameters such as pitch, tempo, or volume.

\section{Copyright Statement}

Copyright (C) 2021 by the Society for Music Theory. All rights reserved.

[1] Copyrights for individual items published in Music Theory Online (MTO) are held by their authors. Items appearing in MTO may be saved and stored in electronic or paper form, and may be shared among individuals for purposes of scholarly 
research or discussion, but may not be republished in any form, electronic or print, without prior, written permission from the author(s), and advance notification of the editors of MTO.

[2] Any redistributed form of items published in MTO must include the following information in a form appropriate to the medium in which the items are to appear:

This item appeared in Music Theory Online in [VOLUME \#, ISSUE \#] on [DAY/MONTH/YEAR]. It was authored by [FULL NAME, EMAIL ADDRESS], with whose written permission it is reprinted here.

[3] Libraries may archive issues of MTO in electronic or paper form for public access so long as each issue is stored in its entirety, and no access fee is charged. Exceptions to these requirements must be approved in writing by the editors of $M T O$, who will act in accordance with the decisions of the Society for Music Theory.

This document and all portions thereof are protected by U.S. and international copyright laws. Material contained herein may be copied and/or distributed for research purposes only.

Prepared by Fred Hosken, Editorial Assistant 